Proyecriones

Vol. 14, $\mathrm{N}^{\circ} 2$, pp. 75-81, December 1995.

Universidad Católica del Norte

Antofagasta - Chile

\title{
A COMMUTATOR RIGIDITY FOR KLEINIAN GROUPS*
}

\author{
Rubén A. Hidalgo \\ Universidad Técnica Federico Santa María, Valparaíso - Chile
}

\begin{abstract}
In these notes we present a particular rigidity property for finitely generated discrete groups of isometries of the hyperbolic three space $\mathbf{H}^{3}$. This property is closely related to the following result obtained in the papers [5], [2], [3] and [4].
\end{abstract}

"Partially supported by Fondecyt 1950830 and Universidad Técnica Federico Santa Maria 951221. 
Theorem 1. Let $G$ be a torsion-free, non-abelian discrete group of isometries of $\mathbf{H}^{3}$. If $G$ is either a

(a) B-group, or

(b) geometrically finite group isomorphic to a fuchsian one,

then $G$ is uniquely determined by $[G, G]$.

The importance of the above in the theory of Kleinian groups concerns Torelli's theorem, which states that a closed Riemann surface is totally determined by its Jacobian variety. This can be considered an step in developing similar results for Riemann surfaces which are not necessarily compact ones. In the language of hyperbolic three manifolds the theorem above asserts that for certain type of 3-manifolds its hyperbolic structure is uniquely determined (up to isometries) by the hyperbolic structure of its homological covering.

It would be important to have a result as above for all finitely generated torsion-free non-abelian Kleinian groups. The property we present in this paper points toward that direction.

Let $\Gamma_{g}$ be a free group of rank $g \geq 2$. Choose free generators $\gamma_{1}, \ldots, \gamma_{g}$, and denote by $\operatorname{Hom}^{*}\left(\Gamma_{g} ; \operatorname{PSL}(2, \mathbf{C})\right)$ the set of homomorphisms $\phi: \Gamma_{g} \rightarrow \operatorname{PSL}(2, \mathrm{C})$ such that

(1) $\phi\left(\Gamma_{g}\right)$ is torsion-free discrete group, and

(2) $\phi\left(\gamma_{1} \gamma_{j} \gamma_{1}^{-1} \gamma_{j}^{-1}\right) \neq I$, for all $j \in\{2, \ldots, g\}$.

Theorem 2 (Main Result). Let $\phi_{1}, \phi_{2} \in \operatorname{Hom}^{*}\left(\Gamma_{g} ; P S L(2, \mathbf{C})\right)$. If $\phi_{1}$ and $\phi_{2}$ coincide on the commutator subgroup $\left[\Gamma_{g}, \Gamma_{g}\right]$, then $\phi_{1}=\phi_{2}$.

Corollary. Let $\Gamma_{g}$ be a free group of rank $g \geq 2$ and let $\phi: \Gamma_{g} \rightarrow \operatorname{PSL}(2, \mathbf{C})$ be a homomorphism such that $\phi\left(\Gamma_{g}\right)$ is a non-abelian, torsion-free discrete group. Then $\phi$ is uniquely determined by its restriction to the commutator subgroup $\left[\Gamma_{g}, \Gamma_{g}\right]$. 
Remark. It is important to note that the main theorem fails if we replace $P S L(2, \mathrm{C})$, the group of orientation preserving isometries of $\mathbf{H}^{3}$, by the group of orientation preserving isometries of a higher dimensional hyperbolic space, say $\mathbf{H}^{n}(n \geq 4)$. In [3] we have constructed an example of two different embeddings of a free group of same rank in the group of orientation preserving isometries of $\mathbf{H}^{4}$, both of them with the same commutator subgroup.

\section{Auxiliary Results}

The following two classical lemmas are basic in the theory of Kleinian groups and they can be found in any book on Kleinian groups (see for example [1] and [6]).

Lemma 1. Let $A, B \in S L(2, \mathrm{C})$. Then

(1) $\operatorname{tr} A^{-1}=\operatorname{tr} A$.

(2) $\operatorname{tr} A B=\operatorname{tr} B A=\operatorname{tr} A \operatorname{tr} B-\operatorname{tr} A^{-1} B$.

(3) $\operatorname{tr} A^{2}=\operatorname{tr}^{2} A-2$.

(4) $\operatorname{tr} A B A^{-1} B^{-1}=-2-\operatorname{tr} A \operatorname{tr} B \operatorname{tr} A B+t^{2} A+\operatorname{tr}^{2} B+\operatorname{tr}^{2} A B$.

(5) $\operatorname{tr} A B^{2} A^{-1} B^{-2}=-2-\operatorname{tr} A\left(\operatorname{tr}^{2} B-2\right)(\operatorname{tr} B \operatorname{tr} A B-\operatorname{tr} A)+\operatorname{tr}^{2} A$ $+\left(\operatorname{tr}^{2} B-2\right)^{2}+(\operatorname{tr} B \operatorname{tr} A B-\operatorname{tr} A)^{2}$.

(6) $\operatorname{tr} A^{2} B A^{-2} B^{-1}=-2-\operatorname{tr} B\left(\operatorname{tr}^{2} A-2\right)(\operatorname{tr} A \operatorname{tr} A B-\operatorname{tr} B)+\operatorname{tr}^{2} B$ $+\left(\operatorname{tr}^{2} A-2\right)^{2}+(\operatorname{tr} A \operatorname{tr} A B-\operatorname{tr} B)^{2}$.

(7) $\operatorname{tr} A B^{-1}=\operatorname{tr} A \operatorname{tr} B-\operatorname{tr} A B$.

In the above, parts (4), (5), (6) and (7) are direct consequences of the above ones. Parts (1), (2) and (3) are direct computations.

Lemma 2. Let $A, B \in S L(2, \mathrm{C})$ be such that $A B-B A \neq 0$. Then $(\operatorname{tr} A, \operatorname{tr} B, \operatorname{tr} A B)$ uniquely determines $A$ and $B$ up to conjugation. Moreover, $(\operatorname{tr} A, \operatorname{tr} B, \operatorname{tr} A B) \neq( \pm 2, \pm 2, \pm 2)$, where the number of minuses is even. 
Theorem 3. Let $A, B \in S L(2, \mathrm{C})$ be such that $A B-B A \neq 0$ and the group $H$ generated by them is a torsion-free discrete group of $P S L(2, \mathbf{C})$. Set $a=\operatorname{tr} A B A^{-1} B^{-1}, b=\operatorname{tr} A B^{2} A^{-1} B^{-2}$ and $c=\operatorname{tr} A^{2} B A^{-2} B^{-1}$. Then $H$ is uniquely determined up to conjugation by the triple $(a, b, c)$.

Proof. Let $H$ be the group generated by $A$ and $B$, which is assumed to be discrete and torsion-free. Set $x=\operatorname{tr} A, y=\operatorname{tr} B$ and $z=\operatorname{tr} A B$. Parts (4), (5) and (6) of lemma 1 imply the following system of equations

$$
\left\{\begin{array}{l}
2+a+x y z-x^{2}-y^{2}-z^{2}=0 \\
2+b+x\left(y^{2}-2\right)(y z-x)-x^{2}-\left(y^{2}-2\right)^{2}-(y z-x)^{2}=0 \\
2+c+y\left(x^{2}-2\right)(x z-y)(x z-y)-y^{2}-\left(x^{2}-2\right)^{2}-(x z-y)^{2}=0
\end{array}\right.
$$

We claim that $a \neq 2$. In fact, assume that $a=2$. Direct computations show that $b=c=2$ and

$$
z=\frac{x y+\sqrt{\left(x^{2}-4\right)\left(y^{2}-4\right)}}{2} .
$$

Since $A B-B A \neq 0$, we have that $A B A^{-1} B^{-1}$ is a parabolic transformation. The transformation $A B^{2} A^{-1} B^{-2}$ is the identity if and only if $A$ and $B^{2}$ commute. Since $A$ is not elliptic of order two, we have that the fixed points of $A$ and $B^{2}$ are the same. It follows that the fixed points of $A$ and $B$ are the same and, in particular, $A B=B A$ which is a contradiction. In consequence, the transformation $A B^{2} A^{-1} B^{-2}$ is parabolic. Similarly, the transformation $A^{2} B A^{-2} B^{-1}$ is parabolic.

Let us consider the following situations.

(1) $x, y \notin\{ \pm 2\}$. In this case we can assume (up to conjugation)

$$
A=\frac{1}{2}\left(\begin{array}{cc}
x & -X \\
-X & x
\end{array}\right) \quad \text { and } \quad B=\frac{1}{2}\left(\begin{array}{cc}
y & -Y r \\
\frac{-Y}{r} & y
\end{array}\right)
$$

where $X^{2}=x^{2}-4, Y^{2}=y^{2}-4$, and $r^{2} \neq 1$. The equality $a=2$ implies $X Y=0$ a contradiction.

(2) If $x \in\{ \pm 2\}$. We may assume $x=2$ and (up to conjugation)

$$
A=\left(\begin{array}{cc}
1 & 1 \\
0 & 1
\end{array}\right) \quad \text { and } \quad B=0\left(\begin{array}{cc}
r & s \\
t & u
\end{array}\right)
$$


where $r u-s t=1$. The equality $a=2$ implies that $t=0$. In this case, the transformation $B$ fixes $\infty$, which is also fixed by the parabolic transformation $A$. Then either $A B=B A$ or the group $H$ is not discrete (in both cases a contradiction).

(3) The case $y \in\{ \pm 2\}$ is similar to (2).

We have now that $a \neq 2$ implies $b \neq 2, c \neq 2$ and

$$
\begin{gathered}
x= \pm \frac{\sqrt{2-c}}{\sqrt{2-a}}, \\
y= \pm \frac{\sqrt{2-b}}{\sqrt{2-a}}, \\
z \in\left\{z_{ \pm}=\frac{1}{2}\left(x y \pm \sqrt{8-4 a-4 x^{2}-4 y^{2}+x^{2} y^{2}}\right)\right\} .
\end{gathered}
$$

Assume that $\left(x, y, z_{+}\right)$determines (up to conjugation) $A$ and $B$. Then as a consequence of lemma 1 , we have that

(1) $\left(-x,-y, z_{+}\right)$determines $-A$ and $-B$.

(2) $\left(-x, y, z_{-}\right)$determines $-A$ and $B$.

(3) $\left(x,-y, z_{-}\right)$determines $A$ and $-B$.

(4) $\left(x, y, z_{-}\right)$determines $A$ and $B^{-1}$.

(5) $\left(-x,-y, z_{-}\right)$determines $-A$ and $-B^{-1}$.

(6) $\left(-x, y, z_{+}\right)$determines $-A$ and $B^{-1}$.

(7) $\left(x,-y, z_{+}\right)$determines $A$ and $-B^{-1}$.

In brief, $a, b$ and $c$ determine $H$ up to conjugation.

\section{Rigidity Property}

In this section, we prove theorem 2. Let $\Gamma_{g}$ be a free group of rank $g \geq 2$. Choose free generators $\gamma_{1}, \ldots, \gamma_{g}$, and denote by $\operatorname{Hom}^{*}\left(\Gamma_{g} ; \operatorname{PSL}(2, \mathbf{C})\right)$ the set of homomorphisms $\phi: \Gamma_{g} \rightarrow \operatorname{PSL}(2, \mathbf{C})$ such that

(1) $\phi\left(\Gamma_{g}\right)$ is torsion-free discrete group, and

(2) $\phi\left(\gamma_{1} \gamma_{j} \gamma_{1}^{-1} \gamma_{j}^{-1}\right) \neq I$, for all $j \in\{2, \ldots, g\}$.

Theorem 2. Let $\phi_{1}, \phi_{2} \in \operatorname{Hom}^{*}\left(\Gamma_{g} ; \operatorname{PSL}(2, \mathbf{C})\right)$. If $\phi_{1}$ and $\phi_{2}$ coincide on the commutator subgroup $\left[\Gamma_{g}, \Gamma_{g}\right]$, then $\phi_{1}=\phi_{2}$. 
Proof. Let $\phi \in \operatorname{Hom}^{*}\left(\Gamma_{g} ; \operatorname{PSL}(2, \mathrm{C})\right)$. Consider $\Gamma_{g}^{j}$ the subgroup of $\Gamma_{g}$ generated by $\gamma_{1}$ and $\gamma_{j}$ for $j=2, \ldots, g$. We restrict $\phi$ to such a subgroup (which is free of rank 2). If we show that $\phi$ restricted to $\Gamma_{g}^{j}$ is uniquely determined by its restriction to the commutator subgroup $\left[\Gamma_{g}^{j}, \Gamma_{g}^{j}\right]$, then we obtain our result. In consequence, it is enough to assume $g=2$. From now on, $g=2$.

Set $U=\phi_{1}\left(\gamma_{1}\right)$ and $V=\phi_{1}\left(\gamma_{2}\right)$. Let $A$ and $B$ be elements in $\operatorname{SL}(2, \mathbf{C})$ representatives of $U$ and $V$, respectively. We write $x=\operatorname{tr} A, y=\operatorname{tr} B$ and $z=\operatorname{tr} A B$. The ambiguity in the choice of the representatives $A$ and $B$ corresponds to a change in the sign of $x$ and $y$, respectively. But this does not affect the sign of the representative of any commutator. In particular, the values $a=\operatorname{tr} A B A^{-1} B^{-1}, b=\operatorname{tr} A B^{2} A^{-1} B^{-2}$ and $c=\operatorname{tr} A^{2} B A^{-2} B^{-1}$ are well defined. As a consequence of theorem 3, we have that there is a transformation $T \in P S L(2, \mathbf{C})$ such that $\phi_{2}\left(\gamma_{1}\right)=T U T^{-1}$ and $\phi_{2}\left(\gamma_{2}\right) \in\left\{T V T^{-1}, T V^{-1} T^{-1}\right\}$.

(1) If $\phi_{2}\left(\gamma_{2}\right)=T V T^{-1}$, then for all $\gamma \in\left[\Gamma_{2}, \Gamma_{2}\right]$ we have $\phi_{1}(\gamma)=\phi_{2}(\gamma)=T \phi_{1}(\gamma) T^{-1}$. That is $T$ commutes with all commutators of the group $\phi_{1}\left(\Gamma_{2}\right)$. The fact that $\phi_{1}\left(\Gamma_{2}\right)$ is torsion-free and non-abelian implies that it is non-elementary and, as a consequence, $T$ must be the identity. It follows then the equality of the homomorphisms in this situation.

(2) If $\phi_{2}\left(\gamma_{2}\right)=T V^{-1} T^{-1}$, then the equality of $\phi_{1}$ and $\phi_{2}$ at the commutator $\gamma_{1} \gamma_{2} \gamma_{1}^{-1} \gamma_{2}^{-1}$ implies $T U V^{-1} U^{-1} V T^{-1}=U V U^{-1} V^{-1}$. In particular, we have the relation

$$
a=t r U V U^{-1} V^{-1}=t r T U V^{-1} U^{-1} V T^{-1}=t r U V^{-1} U^{-1} V
$$

In particular, we have from lemma 1, the following:

$a=\operatorname{tr} U V^{-1} U^{-1} V=\operatorname{tr} U\left(V^{-1} U^{-1} V\right)=\operatorname{tr} U \operatorname{tr} V^{-1} U^{-1} V-t r U^{-1} V^{-1} U^{-1} V$ $=x^{2}-\operatorname{tr}\left(U^{-1} V^{-1} U^{-1}\right) V=x^{2}-\left(\operatorname{tr} U^{-1} V^{1} U^{-1} \operatorname{tr} V-\operatorname{tr} U V U V\right)$ $=x^{2}-y^{2}+z^{2}-2$, that is, we obtain the equality $a=x^{2}-y^{2}+z^{2}-2$. If we combine this with the first equation in the system (1), then we obtain $z=\frac{2 y}{x}$. But in this case, we have a unique $z$ determined, that is, $z_{+}=z_{-}$. In this situation, we have $T V T=T V^{-1} T$, that is, $V$ is elliptic of order two, a contradiction. 


\section{References}

[1] A.F. Beardon. The geometry of discrete groups. Springer-Verlag, Graduate Text in Mathematics, New York, Heidelberg and Berlin, Vol. 91, (1983).

[2] R.A. Hidalgo. Homology coverings of Riemann surfaces. Tôhoku Math. J. 45, pp. 499-503, (1993).

[3] R.A. Hidalgo. Kleinian groups with common commutator subgroup. To appear in Complex Variables.

[4] R.A. Hidalgo. On noded fuchsian groups. Preprint.

[5] B. Maskit. The Homology covering of a Riemann surface. Tôhoku Math. $J .$, pp. 561-562, (1986).

[6] M. Seppälä. Geometry of Riemann surfaces and Teichmüller spaces. North-Holland Mathematics Studies, (1993).

Received : April 02, 1995.

Rubén A. Hidalgo

Departamento de Matemáticas

Universidad Técnica Federico Santa María

Casilla 110-V

Valparaíso - Chile 